\title{
Manipulating solute nucleophilicity with room temperature ionic liquids.
}

\section{Lorna Crowhurst, N. Llewellyn Lancaster, Juan M. Pérez Arlandis and Tom Welton* Electronic Supplimentary Information}

\section{1-Butyl-1-methylpyrrolidinium chloride, [bmpy]Cl}

In a Schlenk flask, 1-chlorobutane $\left(221 \mathrm{~cm}^{3}, 2.05 \mathrm{~mol}\right)$ was added slowly with cooling to 1 methylpyrrolidine $\left(200 \mathrm{~cm}^{3}, 1.92 \mathrm{~mol}\right)$ in propan-2-ol $\left(200 \mathrm{~cm}^{3}\right)$. The mixture was then brought to reflux for 24 hours. After cooling, the solvent was decanted to leave transparent crystals, which were re-crystallized with propan-2-ol, washed with ethyl acetate and then dried under vacuum, giving colourless crystals (314 g, $1.76 \mathrm{~mol}, 92 \%) .{ }^{1} \mathrm{H}-\mathrm{NMR}\left(270 \mathrm{MHz}, \mathrm{DMSO}-d^{6}\right)$ $\delta_{\mathrm{H}}(\mathrm{ppm}): 3.49-3.42(\mathrm{~m}, 4 \mathrm{H}), 3.42-3.34(\mathrm{~m}, 2 \mathrm{H}), 3.02(\mathrm{~s}, 3 \mathrm{H}), 2.06$ (br. s, 4H), 1.66 (quintet, $2 \mathrm{H}, J=7.8 \mathrm{~Hz}), 1.29$ (sextet, $2 \mathrm{H}, J=7.8 \mathrm{~Hz}), 0.91(3, \mathrm{t}, 3 \mathrm{H}, J=7.8 \mathrm{~Hz}) . \mathrm{m} / z(\mathrm{FAB}+): 319$ $\left(\left[(\mathrm{bmpy}){ }_{2} \mathrm{Cl}\right]^{+}, 15 \%\right), 142\left([\mathrm{bmpy}]^{+}, 100 \%\right) \cdot \mathrm{m} / z$ (FAB-): 212 ([(bmpy)Cl $\left.\left.]_{2}\right]^{-}, 100 \%\right), 37$ $\left(\left[{ }^{37} \mathrm{Cl}\right]^{-}, 34 \%\right), 35\left(\left[{ }^{35} \mathrm{Cl}\right]^{-}, 40 \%\right)$. Found: $\mathrm{C}, 60.7 ; \mathrm{H}, 11.6 ; \mathrm{N}, 7.6 . \mathrm{C}_{9} \mathrm{H}_{20} \mathrm{ClN}$ requires: $\mathrm{C}$, $60.8 ; \mathrm{H}, 11.3 ; \mathrm{N}, 7.9 \%$

\section{1-Butyl-1-methylpyrrolidinium bis-(trifluoromethylsulfonyl)imide, [bmpy][N(Tf) $\left.{ }_{2}\right]$} In a Schlenk flask, a solution of [bmpy][N(Tf $\left.)_{2}\right](35.8 \mathrm{~g}, 0.202 \mathrm{~mol})$ in dichloromethane (50 $\mathrm{cm}^{3}$ ) was added to lithium bis-(trifluoromethylsulfonyl)imide (57.4 $\left.\mathrm{g}, 0.200 \mathrm{~mol}\right)$. The resulting suspension was stirred for 72 hours, then filtered. The residual salt was washed with further aliquots of dichloromethane and the combined organic extracts were washed with water until the aqueous phase was halide free (by silver nitrate test), after which the solvent was removed in vacuo. The resulting liquid was treated with activated charcoal and filtered through a pad of acidic alumina to give a colourless liquid (73 g, $0.17 \mathrm{~mol}, 86 \%)$.

${ }^{1} \mathrm{H}-\mathrm{NMR}\left(270 \mathrm{MHz}, \mathrm{DMSO}-d^{6}\right) \delta_{\mathrm{H}}(\mathrm{ppm}): 3.65-3.32(\mathrm{~m}, 4 \mathrm{H}), 3.30-3.24(\mathrm{~m}, 2 \mathrm{H}), 2.96(\mathrm{~s}$, 3H), 2.08 (br. s, 4H), 1.65 (quintet, $2 \mathrm{H}, J=7.4 \mathrm{~Hz}$ ), 1.30 (sextet, 2H, $J=7.4 \mathrm{~Hz}$ ), 0.93 (3, t, 
$3 \mathrm{H}, J=7.4 \mathrm{~Hz}) \cdot m / z(\mathrm{FAB}+): 564\left(\left[(\mathrm{bmpy})_{2}\left(\mathrm{~N}(\mathrm{Tf})_{2}\right)\right]^{+}, 1 \%, 142\left([\mathrm{bmpy}]^{+}, 100 \%\right) \cdot \mathrm{m} / z\right.$

(FAB-): $702\left(\left[(\mathrm{bmpy})\left(\mathrm{N}(\mathrm{Tf})_{2}\right)_{2}\right]^{-}, 5 \%\right), 280\left(\left[\mathrm{~N}(\mathrm{Tf})_{2}\right]^{-}, 100 \%\right)$. Found: C, 31.5; H, 4.7; N, 6.6. $\mathrm{C}_{11} \mathrm{H}_{20} \mathrm{~F}_{6} \mathrm{~N}_{2} \mathrm{O}_{4} \mathrm{~S}_{2}$ requires: $\mathrm{C}, 31.3 ; \mathrm{H}, 4.8 ; \mathrm{N}, 6.6 \%$.

\section{1-Butyl-1-methylpyrrolidinium triflate, [bmpy][OTf]}

In a Schlenk flask, a solution of [bmpy]Cl $(56.0 \mathrm{~g}, 0.315 \mathrm{~mol})$ in dichloromethane $(75 \mathrm{~cm} 3)$ was added to lithium trifluoromethanesulfonate $(49.0 \mathrm{~g}, 0.314 \mathrm{~mol})$. The resulting suspension was stirred for $72 \mathrm{~h}$ and then filtered. The residual salt was washed with further aliquots of dichloromethane, and the combined organic extracts were washed with aliquots of water until the aqueous phase was halide free (by silver nitrate test), after which the solvent was removed in vacuo. The resulting liquid was treated with activated charcoal and filtered through a pad of acidic alumina to give a colorless liquid (67.7 g, $0.23 \mathrm{~mol}, 74 \%)$.

${ }^{1} \mathrm{H}-\mathrm{NMR}\left(500 \mathrm{MHz}, \mathrm{DMSO}-d^{6}\right.$ ) $\delta_{\mathrm{H}}$ (ppm): 3.48-3.39 (4H, m), 3.30-3.26 (2H, m), 2.97 (3H, s), $2.07(4 \mathrm{H}$, br. s), $1.67(2 \mathrm{H}, \mathrm{m}), 1.31(2 \mathrm{H}, \mathrm{m})$, and $0.92(3 \mathrm{H}, \mathrm{t})$.

${ }^{13} \mathrm{C}-\mathrm{NMR}\left(126 \mathrm{MHz}\right.$, DMSO- $d^{6}$ ) $\delta_{\mathrm{C}}(\mathrm{ppm}) 63.37$ (s), 62.87 (s), 47.50 (s), 24.83 (s), 21.03 (s), 19.21 (s) and13.34 (s). MS (FAB $\left.{ }^{+}\right): \mathrm{m} / \mathrm{z} 433$ ([(bmpy) $\left.\left.)_{2} \mathrm{OSO}_{2} \mathrm{CF}_{3}\right]^{+}, 1.3 \%\right)$ and 142 ([bmpy $]^{+}$, $100 \%)$. MS (FAB-): $\mathrm{m} / \mathrm{z} 440$ ([bmpy( $\left.\left.\left(\mathrm{OSO}_{2} \mathrm{CF}_{3}\right)_{2}\right]^{-}, 8.5 \%\right)$ and $\left.\mathrm{m} / \mathrm{z} 149\left(\left[\mathrm{OSO}_{2} \mathrm{CF}_{3}\right]^{-}\right), 100 \%\right)$. Found: $\mathrm{C}, 41.37 \% ; \mathrm{H}, 7.07 \%$;, $4.69 \% . \mathrm{C}_{20} \mathrm{H}_{20} \mathrm{~F}_{3} \mathrm{NO}_{3} \mathrm{~S}$ requires: $\mathrm{C}, 41.23 \% ; \mathrm{H}, 6.92 \%$; $\mathrm{N}, 4.81 \%$. 
Tables of kinetic data

Table S1: Observed rates of reaction of ${ }^{\mathrm{n}}$ butylamines with methyl $p$-nitrobenzenesulfonate in [bmpy] $\left[\mathrm{N}(\mathrm{Tf})_{2}\right]$ at $25^{\circ} \mathrm{C}$.



Standard deviations in parentheses. ${ }^{\mathrm{b}}$ Not fully soluble at this concentration; this value was not used in the determination of $k_{2}$. $\mathrm{n}_{0}$ of methyl $p$-nitrobenzenesulfonate $4.79 \times 10^{-7} \mathrm{~mol}$. 
Table S2: Observed rates of reaction of "butylamines with methyl $p$-nitrobenzenesulfonate in [bmpy][OTf] at $25^{\circ} \mathrm{C}$.

\begin{tabular}{|c|c|c|c|}
\hline$[\text { amine }]_{0}$ & {$[\mathrm{Me} \mathrm{p}-\mathrm{NBS}]_{0}$} & $10^{3} k_{\mathrm{obs}}$ & $k_{2}$ \\
\hline$/ \mathrm{M}$ & $/ \mathrm{mM}$ & $/ \mathrm{s}^{-1}$ & $/ \mathrm{M}^{-1} \mathrm{~s}^{-1}$ \\
\hline & ${ }^{\mathrm{n}} \mathrm{BuNH}_{2}$ & & \\
\hline 0.00616 & 0.308 & $6.51(0.05)$ & \\
\hline 0.0120 & 0.301 & $11.9(0.1)$ & 0.922 \\
\hline 0.0246 & 0.308 & $23.4(0.6)$ & $(0.005)$ \\
\hline \multirow[t]{2}{*}{0.0308} & 0.309 & $29.3(0.2)$ & \\
\hline & ${ }^{\mathrm{n}} \mathrm{Bu}_{2} \mathrm{NH}$ & & \\
\hline 0.00358 & $0.334^{\mathrm{a}}$ & $3.45(0.06)$ & \\
\hline 0.00710 & 0.303 & $7.13(0.05)$ & 1.04 \\
\hline 0.0108 & $0.335^{\mathrm{a}}$ & $10.6(0.5)$ & $(0.03)$ \\
\hline \multirow[t]{2}{*}{0.0143} & 0.305 & $14.7(0.2)$ & \\
\hline & ${ }^{\mathrm{n}} \mathrm{Bu}_{3} \mathrm{~N}$ & & \\
\hline 0.00253 & 0.305 & $0.973(0.008)$ & \\
\hline 0.00505 & 0.305 & $2.43(0.02)$ & 0.523 \\
\hline 0.00765 & 0.305 & $3.65(0.04)$ & $(0.031)$ \\
\hline 0.0151 & 0.305 & $5.40(0.06)^{b}$ & \\
\hline
\end{tabular}

Standard deviations in parentheses. $\mathrm{n}_{0}$ of methyl $p$-nitrobenzenesulfonate $5.06 \times 10^{-7} \mathrm{~mol}$ except ${ }^{\mathrm{a}} \mathrm{n}_{0}=5.52 \times 10^{-7} \mathrm{~mol}^{\mathrm{b}}$ Not fully soluble at this concentration; this value was not used in the determination of $k_{2}$. 
Table S3: Observed rates of reaction of ${ }^{\mathrm{n}}$ butylamines with methyl $p$-nitrobenzenesulfonate in [bmim][OTf] at $25^{\circ} \mathrm{C}$

\begin{tabular}{|c|c|c|c|}
\hline$[\text { amine }]_{0}$ & {$[\mathrm{Me} \mathrm{p}-\mathrm{NBS}]_{0}$} & $10^{3} k_{\mathrm{obs}}$ & $k_{2}$ \\
\hline$/ \mathrm{M}$ & $/ \mathrm{mM}$ & $/ \mathrm{s}^{-1}$ & $/ \mathrm{M}^{-1} \mathrm{~s}^{-1}$ \\
\hline & ${ }^{\mathrm{n}} \mathrm{BuNH}_{2}$ & & \\
\hline 0.0132 & 0.300 & $6.64(0.11)$ & \\
\hline 0.0200 & 0.304 & $10.3(0.2)$ & 0.500 \\
\hline 0.0266 & 0.302 & $13.7(0.2)$ & $(0.016)$ \\
\hline \multirow[t]{2}{*}{0.0327} & 0.297 & $16.3(0.3)$ & \\
\hline & ${ }^{\mathrm{n}} \mathrm{Bu}_{2} \mathrm{NH}$ & & \\
\hline 0.00387 & $0.207^{\mathrm{a}}$ & $1.88(0.02)$ & \\
\hline 0.00747 & $0.261^{\mathrm{a}}$ & $3.90(0.03)$ & 0.541 \\
\hline 0.0115 & 0.298 & $6.00(0.04)$ & $(0.005)$ \\
\hline \multirow[t]{2}{*}{0.0159} & 0.309 & $8.43(0.07)$ & \\
\hline & ${ }^{\mathrm{n}} \mathrm{Bu}_{3} \mathrm{~N}$ & & \\
\hline 0.00273 & $0.270^{\mathrm{a}}$ & $0.172(0.001)$ & \\
\hline 0.00405 & $0.267^{\mathrm{a}}$ & $0.224(0.001)$ & 0.0529 \\
\hline 0.00556 & $0.274^{\mathrm{a}}$ & $0.314(0.001)$ & $(0.0022)$ \\
\hline 0.00831 & $0.273^{\mathrm{a}}$ & $0.462(0.003)$ & \\
\hline
\end{tabular}

Standard deviations in parentheses. $\mathrm{n}_{0}$ of methyl $p$-nitrobenzenesulfonate $4.60 \times 10^{-7}$ mol except ${ }^{\mathrm{a}} \mathrm{n}_{0}=4.14 \times 10^{-7} \mathrm{~mol}$. 
Table S4: Observed rates of reaction of ${ }^{\mathrm{n}}$ butylamines with methyl $p$-nitrobenzenesulfonate in acetonitrile at $25^{\circ} \mathrm{C}$.

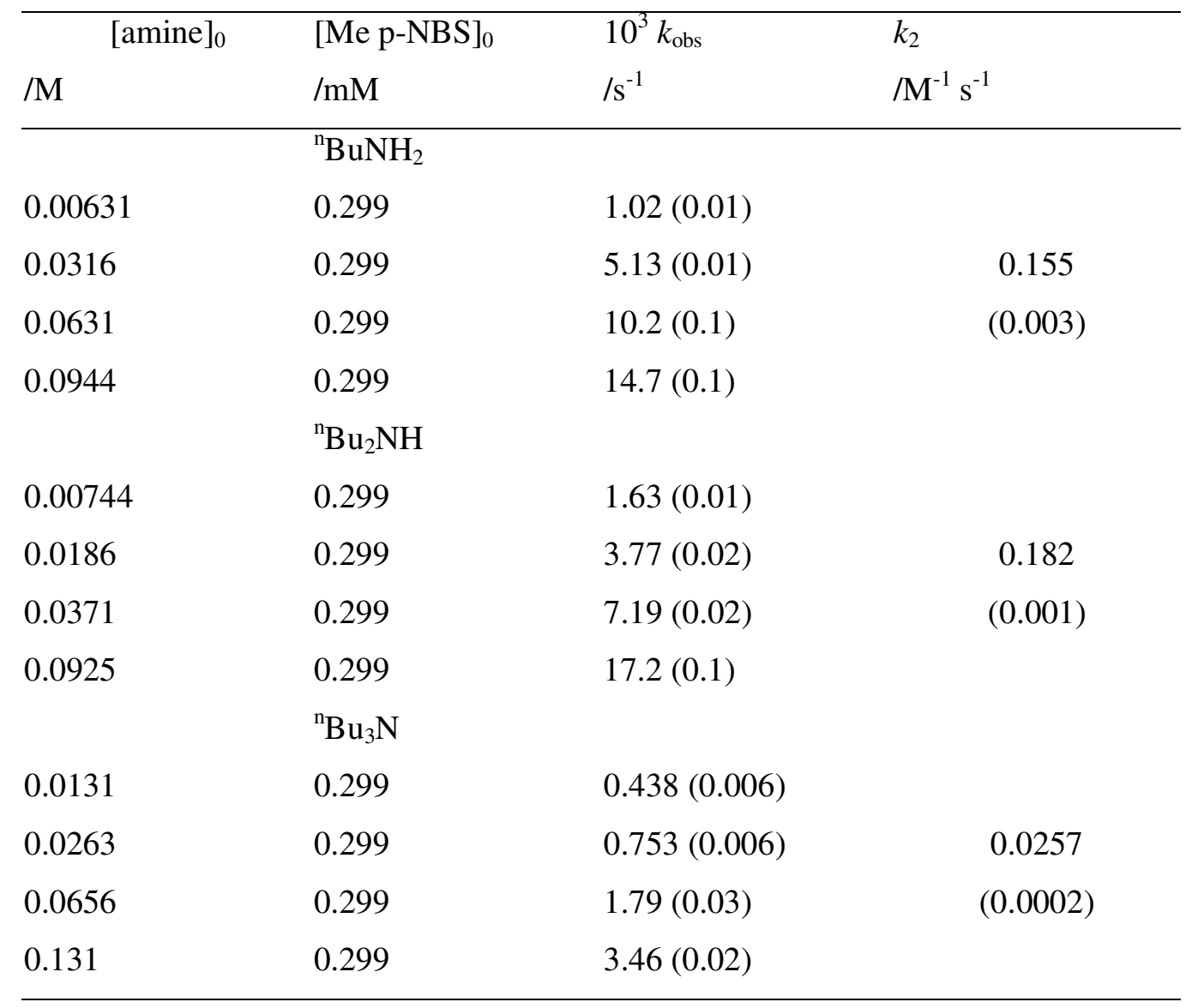

Standard deviations in parentheses. $\mathrm{n}_{0}$ of methyl $p$-nitrobenzenesulfonate $4.79 \times 10^{-7} \mathrm{~mol} ; 1.6$ $\mathrm{cm}^{3}$ total volume. 
Table S5: Observed rates of reaction of ${ }^{\mathrm{n}}$ butylamines with methyl $p$-nitrobenzenesulfonate in dichloromethane at $25^{\circ} \mathrm{C}$.

\begin{tabular}{|c|c|c|c|}
\hline$[\text { amine }]_{0}$ & {$[\mathrm{Me} \mathrm{p}-\mathrm{NBS}]_{0}$} & $10^{3} k_{\mathrm{obs}}$ & $k_{2}$ \\
\hline$/ \mathrm{M}$ & $/ \mathrm{mM}$ & $/ \mathrm{s}^{-1}$ & $/ \mathrm{M}^{-1} \mathrm{~s}^{-1}$ \\
\hline \multicolumn{4}{|c|}{${ }^{\mathrm{n}} \mathrm{BuNH}_{2}$} \\
\hline 0.0316 & 0.299 & $0.600(0.015)$ & \\
\hline 0.0631 & 0.299 & $1.07(0.01)$ & 0.0165 \\
\hline 0.156 & 0.299 & $2.67(0.03)$ & $(0.0001)$ \\
\hline 0.316 & 0.299 & $5.27(0.03)$ & \\
\hline \multicolumn{4}{|c|}{${ }^{\mathrm{n}} \mathrm{Bu}_{2} \mathrm{NH}$} \\
\hline 0.0186 & 0.299 & $0.933(0.019)$ & \\
\hline 0.0371 & 0.299 & $1.80(0.01)$ & 0.0454 \\
\hline 0.0925 & 0.299 & $4.22(0.03)$ & $(0.0002)$ \\
\hline 0.186 & 0.299 & $8.54(0.19)$ & \\
\hline \multicolumn{4}{|c|}{${ }^{\mathrm{n}} \mathrm{Bu}_{3} \mathrm{~N}$} \\
\hline 0.0131 & 0.299 & $0.225(0.011)$ & \\
\hline 0.0263 & 0.299 & $0.572(0.004)$ & 0.0200 \\
\hline 0.0656 & 0.299 & $1.31(0.02)$ & $(0.0005)$ \\
\hline 0.131 & 0.299 & $2.62(0.09)$ & \\
\hline
\end{tabular}

Standard deviations in parentheses. $\mathrm{n}_{0}$ of methyl $p$-nitrobenzenesulfonate $4.79 \times 10^{-7}$ mol; 1.6 $\mathrm{cm}^{3}$ total volume. 
Table S6: Second order rate constants for the reaction of ${ }^{n}$ butylamines with methyl $p$ nitrobenzenesulfonate at varied temperatures in [bmpy][N(Tf $\left.)_{2}\right]$.

\begin{tabular}{cccccc}
\hline [amine $]_{0} / \mathrm{M}$ & $k_{2} / \mathrm{M}^{-1} \mathrm{~s}^{-1}$ & $T / K$ & $\Delta H^{\ddagger} / \mathrm{kJ}$ & $\Delta S^{\ddagger} / \mathrm{J} \mathrm{K}^{-1}$ & $\Delta G^{\ddagger}{ }_{298 \mathrm{~K}} / \mathrm{kJ}$ \\
$\mathrm{mol}^{-1}$ & & & \\
$\mathrm{~mol}^{-1}$
\end{tabular}

The values of $k_{\mathrm{obs}}$ from which the $k_{2}$ values were derived show standard deviations of $4 \%$ or less. Standard deviations in parentheses. 
Table S7: Second order rate constants for the reaction of ${ }^{n}$ butylamines with methyl $p$ nitrobenzenesulfonate at varied temperatures in [bmpy][OTf].

\begin{tabular}{|c|c|c|c|c|c|}
\hline$[\text { amine }]_{0} / \mathrm{M}$ & $k_{2} / \mathrm{M}^{-1} \mathrm{~s}^{-1}$ & $T / K$ & $\begin{array}{c}\Delta H^{\ddagger} / \mathrm{kJ} \\
\mathrm{mol}^{-1}\end{array}$ & $\begin{array}{c}\Delta S^{\ddagger} / \mathrm{J} \mathrm{K}^{-1} \\
\mathrm{~mol}^{-1}\end{array}$ & $\begin{array}{c}\Delta G_{298 \mathrm{~K}}^{\ddagger} / \mathrm{kJ} \\
\mathrm{mol}^{-1}\end{array}$ \\
\hline \multicolumn{6}{|l|}{${ }^{\mathrm{n}} \mathrm{BuNH}_{2}$} \\
\hline 0.0120 & 0.533 & 288.15 & & & \\
\hline 0.0122 & 0.754 & 293.15 & $38.5(2.3)$ & $-116(8)$ & $73.1(6.5)$ \\
\hline 0.0120 & 0.990 & 298.15 & & & \\
\hline 0.0121 & 1.25 & 303.15 & & & \\
\hline \multicolumn{6}{|l|}{${ }^{\mathrm{n}} \mathrm{Bu}_{2} \mathrm{NH}$} \\
\hline 0.00716 & 0.597 & 288.15 & & & \\
\hline 0.00712 & 0.822 & 293.15 & $37.2(2.5)$ & $-120(9)$ & $72.9(6.8)$ \\
\hline 0.00710 & 1.00 & 298.15 & & & \\
\hline 0.00713 & 1.39 & 303.15 & & & \\
\hline \multicolumn{6}{|l|}{${ }^{\mathrm{n}} \mathrm{Bu}_{3} \mathrm{~N}$} \\
\hline 0.00508 & 0.205 & 288.15 & & & \\
\hline 0.00503 & 0.333 & 293.15 & $59.4(2.0)$ & $-51.5(6.7)$ & $74.8(3.8)$ \\
\hline 0.00505 & 0.481 & 298.15 & & & \\
\hline 0.00508 & 0.749 & 303.15 & & & \\
\hline
\end{tabular}


Table S8: Second order rate constants for the reaction of ${ }^{n}$ butylamines with methyl $p$ nitrobenzenesulfonate at varied temperatures in [bmim][OTf].

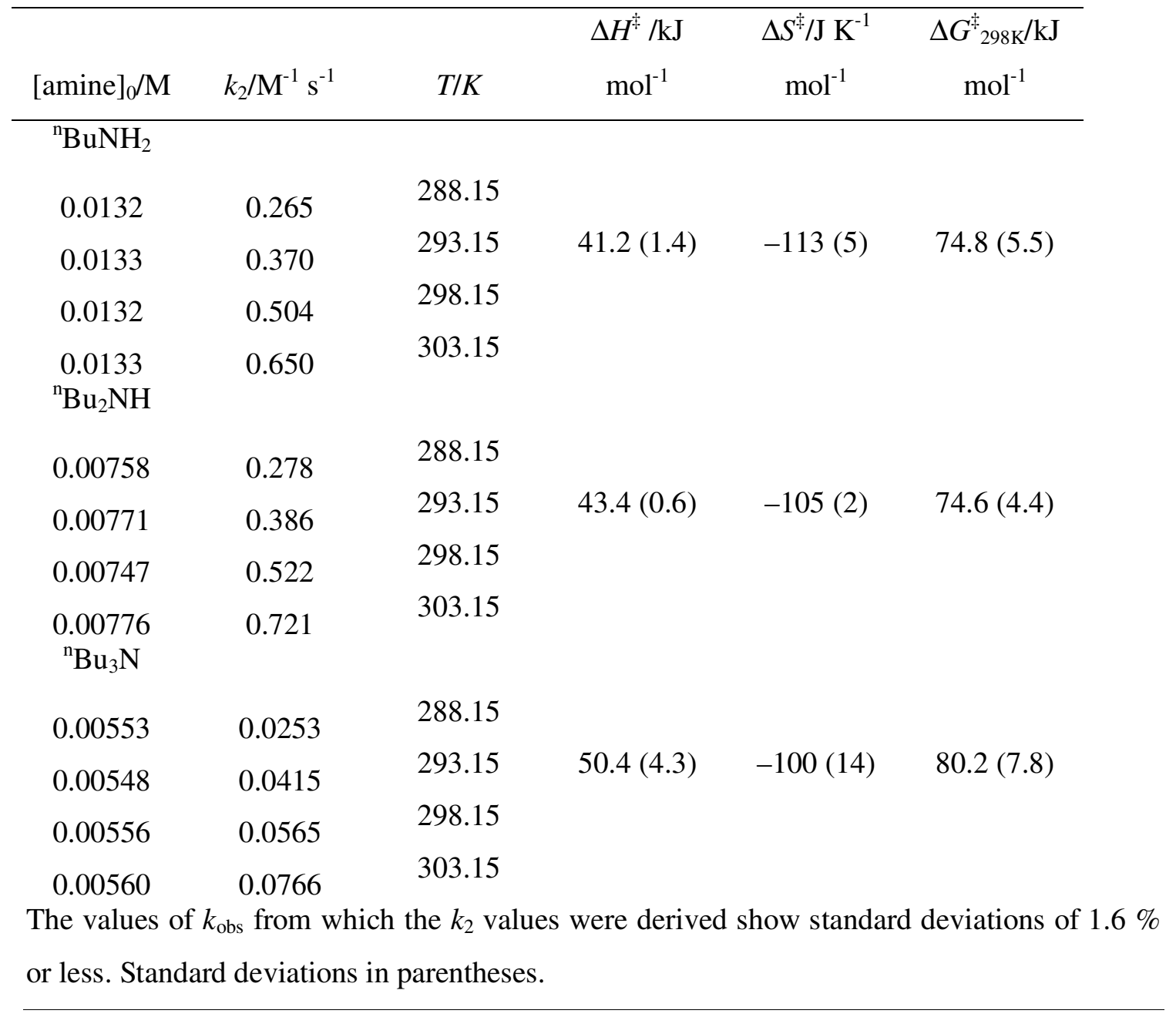


Table S9: Second order rate constants for the reaction of ${ }^{\mathrm{n}}$ butylamines with methyl $p$ nitrobenzenesulfonate at varied temperatures in dichloromethane.

\begin{tabular}{|c|c|c|c|c|c|}
\hline$[\text { amine }]_{0} / \mathrm{M}$ & $k_{2} / \mathrm{M}^{-1} \mathrm{~s}^{-1}$ & $T / K$ & $\begin{array}{c}\Delta H^{\ddagger} / \mathrm{kJ} \\
\mathrm{mol}^{-1}\end{array}$ & $\begin{array}{c}\Delta S^{\ddagger} / \mathrm{J} \mathrm{K}^{-1} \\
\mathrm{~mol}^{-1} \\
\end{array}$ & $\begin{array}{c}\Delta G^{\ddagger}{ }_{298 \mathrm{~K}} / \mathrm{kJ} \\
\mathrm{mol}^{-1}\end{array}$ \\
\hline \multicolumn{6}{|l|}{${ }^{\mathrm{n}} \mathrm{BuNH}_{2}$} \\
\hline 0.0316 & $0.00915^{\mathrm{a}}$ & 288.15 & & & \\
\hline 0.0316 & 0.0127 & 293.15 & & & \\
\hline 0.0316 & 0.0190 & 298.15 & $35.0(6.8)$ & $-162(23)$ & $83.3(13.7)$ \\
\hline 0.0316 & $0.0173^{b}$ & 303.15 & & & \\
\hline 0.0316 & 0.0279 & 308.15 & & & \\
\hline \multicolumn{6}{|l|}{${ }^{\mathrm{n}} \mathrm{Bu}_{2} \mathrm{NH}$} \\
\hline 0.0186 & 0.0331 & 288.15 & & & \\
\hline 0.0186 & 0.0365 & 293.15 & & & \\
\hline 0.0186 & 0.0502 & 298.15 & $32.6(3.1)$ & $-161(11)$ & $80.6(4.6)$ \\
\hline 0.0186 & 0.0629 & 303.15 & & & \\
\hline 0.0186 & 0.0828 & 308.15 & & & \\
\hline \multicolumn{6}{|l|}{${ }^{\mathrm{n}} \mathrm{Bu}_{3} \mathrm{~N}$} \\
\hline 0.0263 & 0.0121 & 288.15 & & & \\
\hline 0.0263 & 0.0156 & 293.15 & & & \\
\hline 0.0263 & 0.0218 & 298.15 & $34.5(2.2)$ & $-161(8)$ & $82.5(4.6)$ \\
\hline 0.0263 & 0.0214 & 300.15 & & & \\
\hline 0.0263 & 0.0268 & 303.15 & & & \\
\hline 0.0263 & 0.0325 & 308.15 & & & \\
\hline
\end{tabular}

The values of $k_{\mathrm{obs}}$ from which the $k_{2}$ values were derived show standard deviations of $4 \%$ or less, except ${ }^{\mathrm{a}}(8 \%)$ and $^{\mathrm{b}}(7 \%)$. 
Table S10: Second order rate constants for the reaction of ${ }^{\mathrm{n}}$ butylamines with methyl $p$ nitrobenzenesulfonate at varied temperatures in acetonitrile.

\begin{tabular}{|c|c|c|c|c|c|}
\hline$[\text { amine }]_{0} / \mathrm{M}$ & $k_{2} / \mathrm{M}^{-1} \mathrm{~s}^{-1}$ & $T / K$ & $\begin{array}{c}\Delta H^{\ddagger} / \mathrm{kJ} \\
\mathrm{mol}^{-1}\end{array}$ & $\begin{array}{c}\Delta S^{\ddagger} / \mathrm{J} \mathrm{K}^{-1} \\
\mathrm{~mol}^{-1}\end{array}$ & $\begin{array}{c}\Delta G^{\ddagger}{ }_{298 \mathrm{~K}} / \mathrm{kJ} \\
\mathrm{mol}^{-1}\end{array}$ \\
\hline \multicolumn{6}{|l|}{${ }^{\mathrm{n}} \mathrm{BuNH}_{2}$} \\
\hline 0.0316 & 0.0832 & 288.15 & & & \\
\hline 0.0316 & 0.109 & 293.15 & & & \\
\hline 0.0316 & 0.162 & 298.15 & $40.0(2.2)$ & $-126(8)$ & $77.6(4.6)$ \\
\hline 0.0316 & 0.200 & 303.15 & & & \\
\hline 0.0316 & 0.257 & 308.15 & & & \\
\hline \multicolumn{6}{|l|}{${ }^{\mathrm{n}} \mathrm{Bu}_{2} \mathrm{NH}$} \\
\hline 0.0186 & 0.105 & 288.15 & & & \\
\hline 0.0186 & 0.143 & 293.15 & & & \\
\hline 0.0186 & 0.203 & 298.15 & $37.5(2.4)$ & $-133(8)$ & $77.1(4.8)$ \\
\hline 0.0186 & 0.245 & 303.15 & & & \\
\hline 0.0186 & 0.308 & 308.15 & & & \\
\hline \multicolumn{6}{|l|}{${ }^{\mathrm{n}} \mathrm{Bu}_{3} \mathrm{~N}$} \\
\hline 0.0263 & 0.0162 & 288.15 & & & \\
\hline 0.0263 & 0.0218 & 293.15 & & & \\
\hline 0.0263 & 0.0286 & 298.15 & $35.7(0.8)$ & $-155(4)$ & $81.9(2.0)$ \\
\hline 0.0263 & 0.0372 & 303.15 & & & \\
\hline 0.0263 & 0.0449 & 308.15 & & & \\
\hline
\end{tabular}

The values of $k_{\mathrm{obs}}$ from which the $k_{2}$ values were derived show standard deviations of $4 \%$ or less. 